\title{
Augmented reality for interpretive and experiential learning
}

\author{
Steven L. Snyder \\ The Franklin Institute \\ 222 North $20^{\text {th }}$ Street \\ Philadelphia, PA 19103 \\ U.S.A. \\ ssnyder@fi.edu
}

\author{
Karen J. Elinich \\ The Franklin Institute \\ 222 North $20^{\text {th }}$ Street \\ Philadelphia, PA 19103 \\ U.S.A. \\ kelinich@fi.edu
}

\begin{abstract}
The Augmented Reality for Interpretive and Experiential Learning (ARIEL) project explores the use of augmented reality in informal science education in the context of a science museum exhibition. The project team has developed an open-source augmented reality (AR) authoring platform designed with the science centre exhibit designer in mind. The platform uses a suite of AR approaches to allow exhibit developers to quickly and inexpensively create augmented interactive experiences. By adding the ability to incorporate real-time modelling and scientific visualisation into the AR experience, the platform acts as a means to use $A R$ as a tool to achieve science centres' educational missions. The project has developed and begun to test a variety of hands-on devices that mix text, images, and visualisations with the kinesthetic experience central to a hands-on approach. This paper will provide an overview of the ARIEL platform and describe a series of interactive device prototypes augmented by the technology along with the results of audience testing and learning research using the devices. ARIEL is a four-year project. This paper presents work to date which is the mid-way point of the project.
\end{abstract}

Augmented reality. Informal science education. Science centre experiences. Interactive device design.

\section{INTRODUCTION}

A central tension within the design of science exhibitions exists between the philosophical commitment to hands-on kinesthetic exploration of phenomena and the need for interpretation of that hands-on exploration. The ideal science exhibit device provides a meaningful primary and sensory involvement with phenomena. To move beyond the primary experience, secondary support - graphic panels, explanatory text, videos, and animations assists the learner in reflecting on the experience. However, this secondary 'interpretive' learning (graphic displays, screens, etc.) is necessarily removed from the actual, hands-on interaction.

Once the device has been used, the learner must make the decision to invest further time and effort to read the graphic panel, make sense of the diagrams, and then return to the primary experience to connect the hands-on and interpretive experience (Falk, 2001). Coupled with reticence to read graphic panels at all (Fleck et al., 2002), this structure makes the interpretation of the hands-on experience a primary challenge when designing interactive exhibit experiences. One approach to the problem of more firmly connecting primary experience and interpretation has been to use augmented reality (AR) systems. Through AR, the primary encounter and secondary interpretation will merge and generate a singular integrated experience.

AR technologies integrate the experiential, interpretive, and social dynamics of the learning experience. Essentially, science museums are constructivist learning environments. Within science museums, intentional learners have countless opportunities to construct knowledge. In contrast, most formal science learning environments - especially K-12 science classrooms - represent science as a discipline for objectivists by presenting science as a body of facts to be known and remembered. Most learners who visit museums are products of those classrooms. Science museums, therefore, need to find the points of intersection between prior objective experience and the invitation to construct new knowledge. AR technology has the potential to enable the connection between the experiential and the interpretive aspects of learning. 


\subsection{Approaches to AR use in Science Centres}

AR systems based in handheld devices are already in regular use in some science museums (Klopfer, 2008), notably in Austria and Japan. For example, The Graz University of Technology in Vienna has developed an innovative handheld AR system called the Museum Augmented Reality Quest (MARQ), which 'aims at developing an electronic tour guide for museums based on a self-contained, inexpensive PDA that delivers a fully interactive 3D AR'(Schmalstieg \& Wagner, 2005).

In Boston, the Museum of Science piloted an AR project developed with the Massachusetts Institute of Technology. The experience, which also used handheld devices, layered a murder mystery over the museum gallery space, challenging participants to locate clues and attempt to solve the mystery. MIT's Teacher Education Program also offers the Developing Public Opinions on Science Using Information Technologies (POSIT) project model, which, again, is handheld-based (Klopfer, 2010). These projects both offer important models, but raise the question of how practical they are for family learning.

AR research (Kondo, et al., 2007) is also underway at the National Science Museum in Tokyo, Japan where a dinosaur gallery is augmented with virtual creatures accessed using a handheld PC. Like the $M A R Q$ and Boston projects, however, the dependence upon handheld devices limits the widespread, practical applicability for science museums.

Another example of AR implementation is CONNECT, a European project that has been using $A R$ as a means for augmenting existing interactive stations with digital visualisations (CONNECT, 2010). Projecting force diagrams and controlled explanatory videos, this system allows visitors to enhance their experience to positive effect. The system was developed specifically for use with visitors from the formal education system. Students strap on a backpack holding the computing platform and wear head-mounted displays. Initial findings from this project demonstrate a definite potential for the approach to improve learning in the science centre setting. Improvements (over control groups) were seen in CONNECT student motivation and learning. While it is too soon to credit this fully to the AR system, the early success indicates that further development of AR interfaces for hands-on devices in the science centre is a potentially fruitful area for exploration.

In all of these examples, however, the reliance upon specialised devices restricts the ability to merge the hands-on experiential with the interpretive. The gear, essentially, becomes the experience. And, the gear effectively forces the learner to learn alone - in contrast to the social learning goals associated with most science exhibits.

All of these examples, however, serve as important pilot efforts that have provided important insights for the field. These projects have informed the Augmented Reality for Interpretive and Experiential Learning (ARIEL) project, which is based at The Franklin Institute Science Museum in Philadelphia, Pennsylvania, U.S.A., which focuses on encouraging a more field-wide exploration of $A R$ technologies and assessing the learning potential of the same by addressing some of the key barriers preventing wider adoption. First and foremost, ARIEL is focused on implementation of site-based AR that does not require any specialised gear. Rather than equipping the visitor with special equipment - such as handhelds, goggles, or backpacks - ARIEL layers the AR enhancements onto the fixed-position exhibit device. Furthermore, the ARIEL project is not just adding AR layers to devices at The Franklin Institute. With funding from the National Science Foundation (ISE \#0741659), ARIEL is building an easy-to-use AR authoring platform using open source software and protocols. The ARIEL platform will be made freely available for use by exhibit designers and is intended to further facilitate experimentation with and adoption of AR technologies by science centres.

\section{AN AUTHORING TOOLKIT}

In order for AR to achieve its full potential in science exhibit design, the steep learning curve associated with AR programming needed to be addressed. For the ARIEL project, The Franklin Institute partnered with the Entertainment Technology Center at Carnegie Mellon University to develop the ARIEL authoring toolkit.

The toolkit is an application suite that consists of a Runner application - which is based on existing open source AR systems - and a Builder which allows a user with limited programming experience to easily construct AR applications. The ARIEL toolkit offers an easy way to prototype AR applications quickly with an eye towards incorporating them into science centre experiences.

Based on existing open source frameworks, the ARIEL toolkit application incorporates four different registration approaches built upon ARToolKit and OpenCV libraries: marker tracking, blob detection/tracking and face recognition. A serial input function based on the openFrameworks library (also used for general rendering, camera functions, etc.) has been added specifically with the 


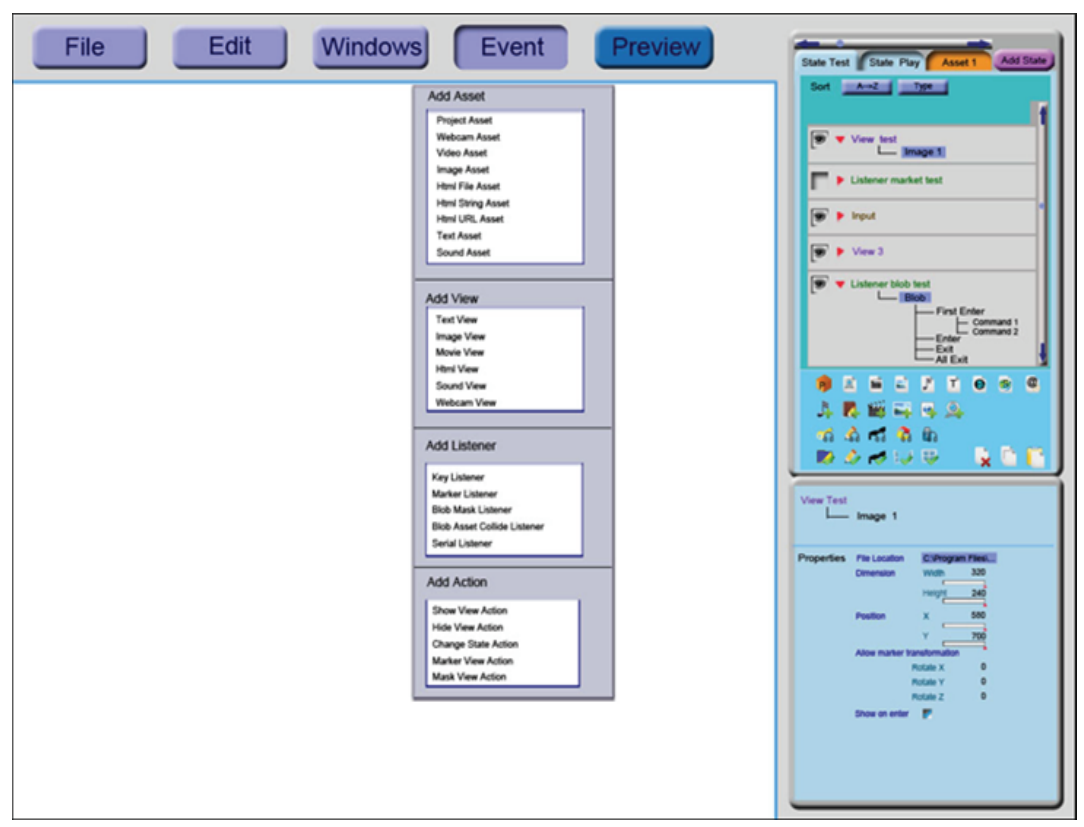

Figure 1: User Interface for ARIEL Builder v1

development of mechanical science centre interactives in mind. Each of these inputs can be then be associated with a standard set of assets (html text, sound files, image files, video and 3D models) allowing the user to create an AR application that uses the selected inputs to manipulate the associated assets.

To create an application the developer accesses the ARIEL builder (Figure 1) a UI based application built upon Nokia's Qt. This interface allows the developer to select assets, associate them with an input method and define a limited set of interactions between them. An arbitrary number of inputs and assets can be assigned. A preview window allows the user to test their application and when completed save the output as an $\mathrm{xml}$ file. This $\mathrm{xml}$ output is then utilised to determine the final operating parameters of the runner application. Both the ARIEL builder and runner have been designed to be easily extensible.

As mentioned above, in addition to the traditional AR registration approaches (markers, background differencing, etc.) a serial input function has been added to ARIEL. This element is very important for a system designed with science centre interactives in mind. The use of markers for example is often ill advised as the hands-on nature of the experience naturally leads to the covering of said markers. Additionally, most interactive device elements are constrained in some way, magnets are attached to pivots, cars roll down tracks, spheres are mounted to armatures, to allow for device reset and to prevent components from straying from their stations. These constraints can then be used to provide registration information when connected to a device like an optical encoder or rheostat.

\subsection{Example prototypes}

The Franklin Institute recently opened two new exhibits: Electricity and Changing Earth. During thedevelopment process, prototype devices were built for these exhibits. Those prototypes also became useful for the ARIEL project. Three of the devices selected for augmentation, evaluation, and research where: 'Be the Path', 'Circuit Bench', and 'Stream Table'.

From the Electricity exhibit, 'Be the Path'(Figure 2) is a device that invites learners to complete an electrical circuit and discover that the human body is a conductor of electricity. A two-foot square kiosk features a flat table top on which there are two light bulbs, two batteries, and four metal spheres. (The spheres are approximately the size of baseballs.) Additionally, thin wires run from the spheres to the battery and bulb. When learners grasp the two metal spheres, they complete the circuit and the bulb lights. It is a quick and easy experience with instant gratification. Most learners smile and run away, satisfied that they lit the bulb.

The real intent of the device, however, is to invite learners to think about the human body as a conductor and to think about electrical circuits. The mounted graphic panel invites experimentation and play in an attempt to demonstrate the need for a complete circuit of conductors. For example, the label copy invites learners to try extending the circuit by linking hands with peers or others nearby. 


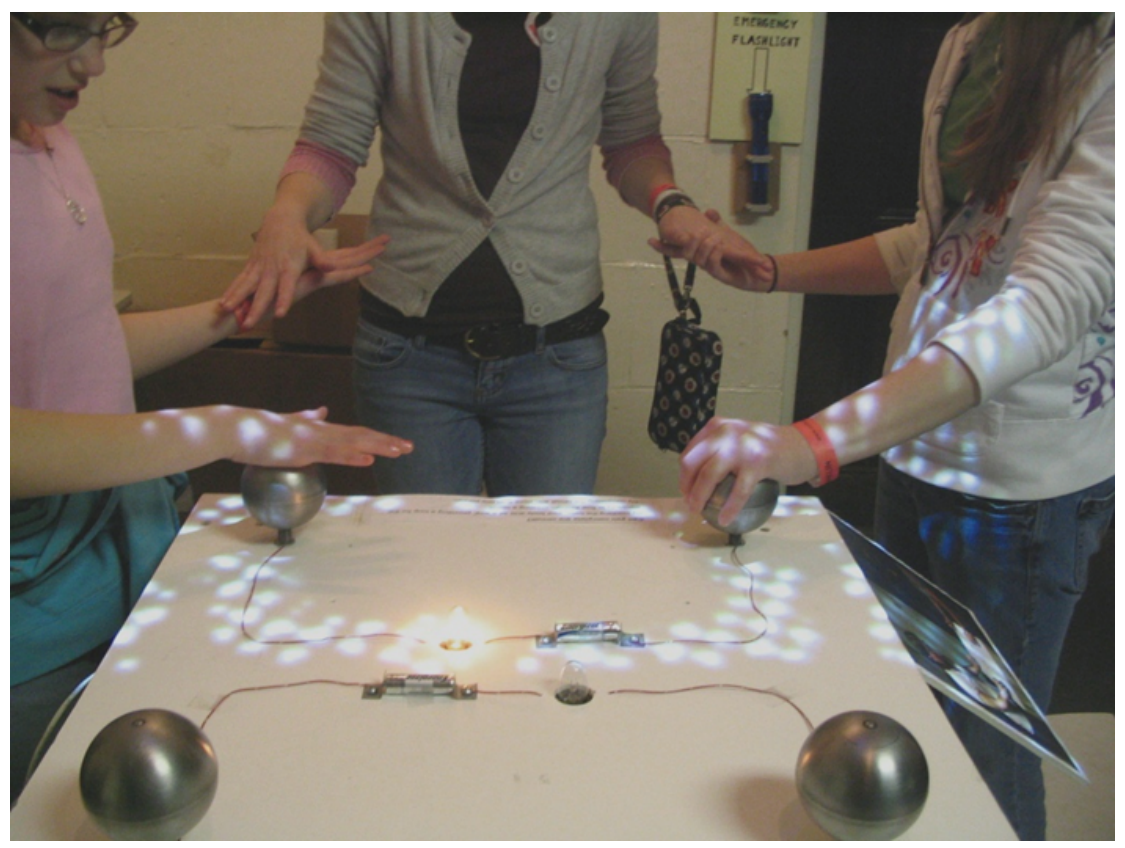

Figure 2: 'Be the Path' uses background differencing to project the flow of electricity when the circuit is complete

When learners ignore the graphic panel, as they often do, they enjoy the instant gratification of the lit bulb, but they miss the point of the device and derive only partial benefit from the experience.

Through ARIEL, 'Be the Path' has been enhanced with a digital projection that uses background differencing to overlay a digital visualisation of a current of flowing electrons onto the body of the learner when the bulb lights. So, the learner grasps the two metal spheres, the bulb lights, and, in the same instant, a projection appears on the learner's arms, shoulders, and head. If the learner lets go of the spheres, the projection vanishes. Grasp the spheres, and the projection reappears and the bulb lights. The intent of the digital augmentation is to draw the learner more fully into interaction with the device. Literally, the projection shows the learners 'being the path'.

Also from the Electricity exhibit, 'Circuit Bench' invites learners to construct electrical circuits of varying complexities. At the bench, visitors find a collection of components that can be assembled into a wide variety of formations. The components include batteries, buzzers, LEDs, switches, motors, and photo-resistors. Each is mounted onto a form that has magnets at each end. By connecting the magnets, the forms merge and, if properly assembled, complete the circuit.

For ARIEL, the 'Circuit Bench' forms include markers (glyphs) that trigger graphic display panels that provide supporting information about each component. Visitors can and do construct successful circuits through open-ended play, but when the marker triggers the added detail, the potential for complexity within their designs increases.

From the Changing Earth exhibit, the 'Stream Table' device prototype was selected for use in the ARIEL project. 'Stream Table' is a fairly classic device in earth science exhibitions. The direct hands-on engagement with a model landscape is a powerful learning experience. A stream of water flows through the landscape, causing change over time. Changes to the landscape, such as the removal of groundcover or construction of new homes, impact the balance between land and water. Likewise, changes to the flow of water, especially the placement or removal of a dam, impacts the balance.

For ARIEL, the models - dams, buildings, trees, and bridges - have been tagged with a marker (glyph) that triggers the projection of real-world video of a comparable landscape. The intent is to help visitors connect their hands-on play with realworld locations and think about the impacts of construction and deforestation on ecosystems.

All three devices - 'Be the Path', 'Circuit Bench', and 'Stream Table' - were designed in consideration of the seven characteristics of familyfriendly exhibit devices. These are: multi-sided family can cluster around exhibit; multi-user interaction allows for several sets of hands (or bodies); accessible - comfortably used by children and adults; multi-outcome - observation and interaction are sufficiently complex to foster group discussion; multi-modal - appeals to different 
learning styles and levels of knowledge; readable text is arranged in easily understood segments; and relevant - provides cognitive links to visitors' existing knowledge and experience (Borun \& Dritsas, 1997). This consideration enables the ARIEL evaluation and research to focus on the impact of the augmented reality apart from concerns about family-friendly learning design. By selecting devices that were already designed to include the family-friendly characteristics, the evaluation and research can focus on the augmentations.

\subsection{Visitor studies}

To date, evaluation and research efforts have focused on 'Be the Path'. Other devices will be evaluated and used in the project's learning research study in the coming months.

Evaluation of 'Be the Path' with the AR feature enabled found that $100 \%$ of the visitors $(n=34)$ succeeded in completing the circuit and activating the AR projection. $61 \%$ of the visitors correctly identified the AR projection as a representation of the flow of electricity through a circuit. $82 \%$ knew that the intent of the device was to use the body's conductivity to complete the flow of electricity through a circuit.

'Be the Path' was also used in the ARIEL project's learning research study in an attempt to determine if the AR projection impacts student understanding of the science content. Therefore, two conditions were established: the use of the device without the AR enhancement (condition 1) and the use of the device with the AR enhancement (condition 2). For the pilot study, 8 middle school students were invited to participate. Half were randomly assigned to each condition.

The first key metric considered was time on task. The hypothesis was that students who encountered the device with the AR enhancement would stay engaged with the device longer and be more likely to experiment. In the pilot study, only one student who encountered ' $\mathrm{Be}$ the Path' without the digital augmentation (condition 1) was engaged beyond the 4-minute mark. In comparison, all of the students who experienced the augmentation (condition 2) stayed engaged for more than 4 minutes and up to 7 minutes. Likewise, students in condition 2 showed a higher level of experimentation, being more likely to attempt to confound the device and figure out other ways to compete the circuit.

While the very small sample size prevents any generalisations from the pilot study, the data does suggest that AR may potentially positively influence visitor engagement with a device and its intent. In the coming months, these ideas will be tested with larger sample populations in the coming months.

\subsection{Next steps}

When the ARIEL project was first imagined, the 'Magnetic Maps' device was a conceptual touchstone. In this familiar device, two mounted bar magnets pivot freely. As visitors rotate each magnet, they feel the attractive and repulsive forces as the poles encounter each other. In the AR experience, an overhead camera captures this activity and, based on the magnets' relative position, calculates and then superimposes a graphic representation of the combined magnetic field. In this way, users both feel and see the interaction between the magnets. The augmented reality effectively 'maps' the magnetic force fields as they intersect, providing a real-time visualisation of the physical phenomena.

Early prototypes of the 'Magnetic Maps' device have had only moderate success as the ability to integrate the necessary real-time modelling into the experience had not yet been perfected. Now, with an established base authoring application, the next step for ARIEL is the development and integration of that real-time modelling into the system.

One of the great strengths of a computer-based augmentation to a mechanical hands-on interactive is the ability to use the processing power of the system to calculate and visualise datasets associated with the phenomena being explored.

The ARIEL toolkit phase-two module will pull location, orientation and velocity information from the chosen ARIEL tracking approach and send that data to a user-defined modelling routine which in turn will generate a visualisation data set to be combined with live video and sent to the chosen output. An initial set of modelling options will focus on displaying real-time scalar, vector, and field data sets. Additionally, the ability to use the state of one registration approach (serial input, for example) to affect the output of another (the scale of an image attached of a marker, for example) will also be included in this next expansion of the system.

It is expected that the fully-operational ARIEL system will be available for public download by late August 2010.

This material is based upon work supported by the National Science Foundation under Grant No. 0741659. Any opinions, findings, and conclusions or recommendations expressed in this material are those of the author(s) and do not necessarily reflect the views of the National Science Foundation. 


\section{REFERENCES}

Borun, M., \& Dritsas, J. (1997) Developing familyfriendly exhibits. Curator, 40(3), pp. 178-196.

CONNECT (2010) The CONNECT Project. http://www.ea.gr/ep/connect/ (12 March 2010)

Falk, J. (2001) Free-Choice Science Education: How We Learn Science Outside of School. Teachers College Press, New York.

Fleck, M., Frid, M., Kindberg, T., O'Brien-Strain, E., Rajani, R., \& Spasojevic, M. (2002) From Informing to Remembering:Ubiquitous Systems in Interactive Museums. IEEE Pervasive Computing 1(2), pp. 13-21.

Klopfer, E. (2008) Augmented learning: research and design of mobile educational games. MIT Press, Cambridge, MA.
Klopfer, E. (2010) Public Opinions of Science using Information Technologies.

http://education.mit.edu/drupal/ar/projects\#posit (12 March 2010)

Kondo, T., Shibasaki, J., Arita-Kikutani, H., Manabe, M., Inaba, R., and Mizuki, A. (2007) Mixed Reality Technology at a Natural History Museum. InMuseums and the Web 2007. http://www.archimuse.com/mw2007/papers/kondo/ kondo.html (12 March 2010)

Schmalstieg, D., and Wagner, D. (2005) A Handheld Augmented Reality Museum Guide. IADIS International Conference on Mobile Learning. http://www.icg.tugraz.ac.at/Members/daniel/Publications/HandheldA R MuseumGuide (12 March 2010) 